\title{
Contribution à la connaissance de la faune d'Oligochètes aquatiques du pays basque et zones limitrophes
}

\author{
P. Rodriguez' \\ J.C. Armas 1
}

Une liste de 59 espèces d'Oligochètes aquatiques appartenant à 5 familles est présentée. Neuf d'entre elles sont nouvelles pour la Péninsule Ibérique. On remarque la présence de Nais christinae et Pristina proboscidea.

A contribution to information on the aquatic oligochaete fauna of the Pays Basque and adjacent areas.

A list of 59 species, belonging to 5 families of aquatic oligochaetes is presented. Nine of them are first records for the Iberian Peninsula. The finding of Nais christinae and Pristina proboscidea is emphasised.

Malgré la situation privilégiée de la Péninsule Ibérique, comme zone de passage entre la Méditerranée et l'Atlantique, l'Afrique et l'Europe continentale, l'étude de la faune benthique de ses fleuves est relativement récente. Ainsi, Margalef (1955) fournit les premières données sur les oligochètes aquatiques. Postérieurement, nous comptons une série de travaux peu nombreux et qui incluent un pet it nombre d'espèces (Hrabe 1963, Haro 1964, Delay 1973, Brinkhurst 1978).

La publication d'une série de travaux récents (Martinez-Ansemil et Giani 1980, Giani et Martinez. Ansemil 1981, Martinez-Ansemil 1981) a élargi considérablement le spectre des espèces connues en Espa. gne. La plupart de ces espèces appartiennent à la faune européenne, mais certaines n'ont été trouvées, jusqu'à présent, qu'en Afrique ou en Amérique (Pristina sima, $P$. osborni et $P$. notopora).

Ce travail effectué sur 5 bassins différents, est un complément à ces études antérieures, au Nord de la Péninsule.

Nous remercions $\mathbf{M}^{r} \mathbf{N}$. Giani (Toulouse) pour son aide dans la détermination ou la confirmation des espèces, nos collègues du laboratoire et $\mathrm{X}$. Iribar qui nous ont fourni les échantillons de plusieurs localités du Pays Basque et du Nord de Burgos.

\footnotetext{
1. Laboratorio de Zoologia, Facultad de Ciencias, Universidad del Pais Vasco Apdo. 644. Bilbao. Espana.
}

\section{1. - Milieux et Méthodes}

Les prélèvements ont été réalisés principalement sur les rivières Butron et Nervion, pendant l'année 1981. Quelques échantillonnages ont été réalisés dans le bassin du Deva (1978 et 1979), de l'Ebro et de l'Oria (1981). Lors d'un prochain travail, nous détaillerons les caractéristiques des bassins et des points d'échantillonnage. La carte de la figure 1 situe les stations que nous avons prospectees. Dans le Nervion, les récoltes ont été réalisées à l'aide d'un filet Surber de $200 \mu \mathrm{m}$ de vide de maille et un cadre de $28 \times 28 \mathrm{~cm}$. Ceci a été possible grâce au type de substrat de la rivière, composé essentiellement par du gravier, des cailloux et de grosses pierres. Mais lorsque le fond du Butron est profond et le substrat argileux et dur, la drague EKMAN n'a pu être utilisée avec succès : dans ces sections il nous a fallu pratiquer les prélèvements avec un flacon directement sur les berges (stations 12 et 13).

\section{2. - Résultats faunistiques}

Nous présentons la liste des espèces trouvées en précisant tout d'abord pour chacune d'elles, le nombre total d'individus determinés (n), puis les citations antérieures pour la Péninsule Ibérique ou d'autres zones d'intérêt, et nous faisons enfin une brève 


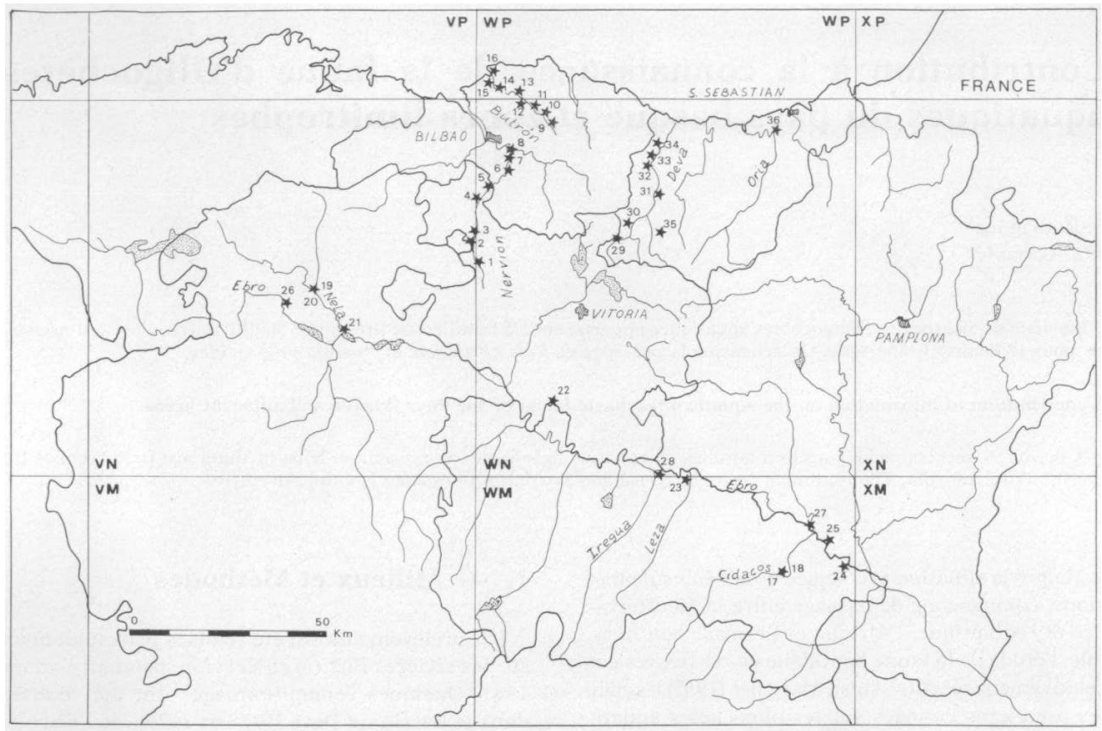

Fig. 1. Carte géographique avec l'emplacement des stations de récolte.

allusion au type d'habitat, à la morphologie ou à la taxonomie, dans les cas oú cela nous semblait intéressant.

\section{- Famille Lumbriculidae}

1. Bythonomus lemani (Grube, 1879) $\mathrm{n}=80$.

Stations: 1, 4, 6, 7, 10.

Péninsule Ibérique : Rodriguez (1981).

2. Lumbriculus variegatus (Muller, 1774) $\mathrm{n}=6$.

Stations : 7, 8, 13, 34, 35.

Péninsule Ibérique : Martinez-Ansemil et Giani (1980).

Remarques : sur les quatre individus sexuellement mûrs, récoltés à la station 13 , trois ont pu ètre étudiés. Les pores mâles sont sur le segment $\mathrm{X}$; les variations dans la composition de l'appareil génital sont présentées sur la figure 2 . Atrium non cylindrique, allongé, pouvant même traverser le dissépiment jusqu'au segment féminin (exemplaire 3). Timm (1979) a souligné la grande variabilité à laquelle est sujette cette espèce ; nos dessins suivent sa nomenclature. La position des pores des spermathèques est normale, dorso-latérale, sous la ligne des soies dorsales. On n'observe en aucun cas le canal déférent et les entonnoirs spermatiques. Le nombre de spermathèques de nos exemplaires peut atteindre 6 . La possibilité d'absence de canaux déférents et d'entonnoirs spermatiques, ainsi que le nombre élevé des spermathèques avaient déjà été signalés par Timm (1979). La présence de 3 paires d'ovaires (exemplaire 2, fig. 2), est à notre connaissance un fait nouveau. Seule avait été décrite la possibilité que, dans un des segments, l'ovaire apparaisse double.

Cook (in Brinkhurst et Jamieson 1971) reprend la description de 5 sous-espèces en fonction de la position du pore mâle et du nombre de paires de testicules et d'ovaires. Pour chaque sous-espece il établit la fréquence d'apparition des différents types; il montre ainsi qu'il existe des chevauchements entre les 5 sous-espèces. Celles-ci se différencieraient prin- 


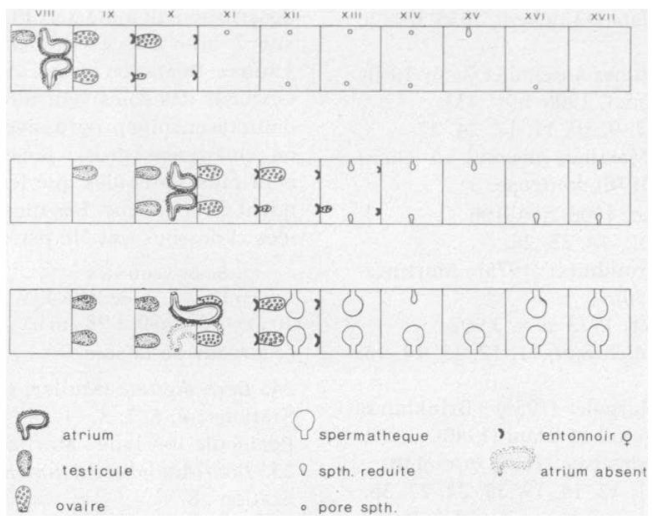

Fig. 2. Schéma de l'appareil génital de plusieurs spécimens de Lumbriculus variegatus. VIII à XVIII : numéro des segments.

cipalement par la plus ou moins grande fréquence d'apparition des différents types dans chaque population. Nos exemplaires présentent les pores mâles en $\mathrm{X}$ et au moins 2 paires d'ovaires ; nous pensons que l'on peut les inclure dans la sous-espece incontans Smith, signalée en Amérique du Nord.

3. Stylodrilus heringianus Claparède, $1862 n=4$. Stations: 1 .

Péninsule Ibèrique : Prat (1980), Martinez-Ansemil et Giani (1980).

4. Stylodrilus parvus (Hrabe et Cernosvitov, 1927) $\mathrm{n}=39$.

Stations : 7, 8, 24.

Péninsule Ibérique : Martinez-Ansemil et Giani (1980).

\section{- Famllle Naididae}

5. Chaetogaster diastrophus (Gruithuisen, 1828) $\mathrm{n}=145$.

Stations: 2, 4, 5, 6, 7, 8, 11, 12, 14, 24, 27, 28.

Péninsule Ibérique: Brinkhurst (1978) douteuse; Martinez-Ansemil et Giani (1980).

6. Chaetogaster diaphanus (Gruithuisen, 1828) $n=$ 386.

Stations: $2,3,4,6,7,8,11,12,13,14,17,18,24$.

Péninsule Ibérique : Rodriguez (1981).
7. Chaetogaster langi Bretscher, $1896 \mathrm{n}=11$.

Stations : 11, 14.

Péninsule Ibérique : Martinez-Ansemil et Giani (1980).

8. Chaetogaster limnaei Von Baer, $1827 \mathrm{n}=8$.

Stations: 10, $12,35$.

Péninsule Ibérique: Alvarez et Selga (1967): Martinez-Ansemil et Giani (1980); Brinkhurst (1978).

9. Amphichaeta sannio Kallstenius, $1892 \mathrm{n}=16$.

Station : 15.

Péninsule Ibérique : Martinez-Ansemil (1981) ; Rodriguez (1981).

10. Amphichaeta leydigii Tauber, $1879 \mathrm{n}=2$.

Station: 24.

Péninsule Ibérique : Martinez-Ansemil et Giani (1980).

11. Paranais frici Hrabe, $1941 \mathrm{n}=23$.

Stations: 4, 7, 13, 15, 25, 27.

Péninsule Ibérique : Rodriguez (1981).

12. Paranais litoralis (Muller, 1784) $\mathrm{n}=403$.

Stations: $2,4,15,16$.

Péninsule Ibérique: Martinez-Ansemil et Giani (1980), dans l'estuaire du Tambre (Galice).

13. Uncinais uncinata ( $\phi \mathrm{rsted}, 1842) \mathrm{n}=10$.

Stations: 11, 12, 14.

Pénirsule Iberique : Martinez-Ansemil et Giani (1980). 14. Ophidonais serpentina (Muller, 1773) $n=45$. Stations: 2, 4, 12, 13, 14, 17.

Péninsule Ibérique: Première donnée. 
15. Slavina appendiculata D'Udekem, $1855 \mathrm{n}=6$. Stations : 11, 14.

Péninsule Ibérique : Martinez-Ansemil et Giani (1980).

16. Nais communis Piguet, $1906 n=433$.

Stations : 1, 2, 4, 5, 6, 7, 9, 10, 11, 12, 24, 27.

Péninsule Ibérique: Martinez-Ansemil et Giani

(1980); Brinkhurst (1978) douteuse

17. Nais pardalis Piguet, $1906 \mathrm{n}=106$.

Stations : 1, 2, 10, 11, 12, 14, 23, 26.

Péninsule Ibérique : Brinkhurst (1978); Martinez-

Ansemil et Giani (1980).

18. Nais elinguis Muller, $1773 \mathrm{n}=3907$.

Stations: 1, 2, 3, 4, 5, 6, 7, 8, 9, 11, 12, 13, 14, 15, $22,23,24,27,36$.

Péninsule Ibérique: Margalef (1955); Brinkhurst

(1978) : Martinez-Ansemil et Giani (1980).

19. Nais bretscheri Michaelsen, $1899 \mathrm{n}=110$

Stations: 1, 6, 8, 10,11, 12, 14, 19, 23, 24, 27, 36.

Péninsule Ibérique : Martinez-Ansemil (1981) ; Rodriguez (1981).

20. Nais pseudobtusa Piguet, $1906 \mathrm{n}=25$.

Stations : 10, 11.

Péninsule Ibérique : Brinkhurst (1978); Martinez-

Ansemil et Giani (1980).

21. Nais alpina Sperber, $1948 \mathrm{n}=98$.

Stations : 10, 11, 26.

Péninsule Ibérique : Martinez-Ansemil et Giani (1980).

22. Nais barbata Muller, $1773 \mathbf{n}=724$.

Stations: $1,2,4,5,6,7,8,9,10,11,12,13,14,17$, 18, 26, 27, 28, 36 .

Péninsule Ibérique : Première donnée.

23. Nais christinae Kasprzak, $1973 \mathrm{n}=5$.

Stations : 6,7 .

Péninsule Ibérique : Première donnée.

Répartition : Pologne (Kasprzak 1973 a) ; Tchécoslovaquie (Hrabe 1981) : France (Lafont et Juget 1976, Lafont 1982).

Remarques: On la trouve sur fond caillouteux, dans des zones de récupération des sections antérieures présentant une forte pollution organique et industrielle.

Hrabe (1981) considère Nais variabilis var. " des grands lacs „ Piguet (1906), citee dans les lacs suisses, et Nais variabilis part., citee par Sperber (1948) en Suède, comme synonymes de Nais christinae Kasprzak. Les soies de nos exemplaires correspondent plus aux dessins de Hrabe qu'à ceux de Kasprzak, dans la largeur des dents des soies ventrales postérieures et dans la forme des dents des aiguilles.
Description du matériel : Présence évidente d'yeux sur 2 individus et faiblement marquee sur les 3 autres. Dilatation stomacale en VII. La plus grande longueur des soies ventrales s'obverve en II ; elle diminue ensuite progressivement de III à V. En VI, on remarque un grossissement des soies qui deviennent plus sigmoïdes que les précédentes et diminuent de longueur. Les mesures des soies présentées ci-dessous ont été prises sur un exemplaire.

$$
\begin{array}{cl}
\text { Soies ventrales } & \text { Soies dorsales } \\
\text { en II de VI à XIV aiguille capillaire }
\end{array}
$$

$110 \mu \mathrm{m}-\mathrm{l}: 3 \mu \mathrm{m} 90$ à $98 \mu \mathrm{m}-1: 5 \mu \mathrm{m} 64.92 \mu \mathrm{m} 250-255 \mu \mathrm{m}$

1 : largeur de la soie.

24. Dero digitata (Muller, 1773) $\mathbf{n}=76$.

Stations : 4, 6, 7, 8 .

Péninsule Ibérique: Martinez-Ansemil (1981).

25. Dero (Aulophorus) furcatus (Muller, 1773) $\mathrm{n}=\mathbf{2}$. Station: 8 .

Péninsule Ibérique : Brinkhurst (1978).

26. Pristina foreli (Piguet, 1906) $\mathrm{n}=5$.

Stations : $10,11,12$.

Péninsule Ibérique : Martinez-Ansemil et Giani (1980).

27. Pristina aequiseta Bourne, $1891 \mathrm{n}=7$.

Stations : 10, 11.

Péninsule Ibérique : Martinez-Ansemil et Giani (1980). Remarques : D'après Harman (1980) et Loden et Harman (1980), cette espèce serait synonyme de P. foreli, avec laquelle elle coexiste dans le Butron.

28. Pristina menoni (Aiyer, 1929) $\mathbf{n}=3$.

Stations: 6,11 .

Péninsule Ibérique : Martinez-Ansemil et Giani (1980). 29. Pristina sima (Marcus, 1944) $\mathrm{n}=5$.

Stations : 4, 10.

Péninsule Ibérique : Martinez-Ansemil et Giani (1980).

Habitat : Eaux peu polluées, sur fonds caillouteux.

Remarques : En accord avec la description faite par Martinez-Ansemil et Giani (1980) nos exemplaires ont les dents latérales des aiguilles inégales, la dent inférieure pouvant atteindre le double de la longueur de la supérieure. Sperber (1948) et Brinkhurst (in Brinkhurst et Jamieson, 1971) n'indiquent pas ce caractère dans leurs descriptions. Sperber signale la similitude de cette espèce avec $P$. notopora et $P$. osborni, desquelles nos exemplaires diffèrent nettement. Nous pensons comme Loden \& Harman (1980) que $P$. sima pourrait etre considérée comme proche du groupe de $P$. rosea.

30. Pristina rosea (Piguet, 1906) $\mathrm{n}=76$.

Stations: $1,2,6,7,9,10,11,12$. 
Péninsule Ibérique : Première donnée.

Remarques : $P$, rose $a$ est largement représentée sur tout le continent européen : cette donnée confirme l'opinion de Martinez-Ansemil et Giani (sous presse) sur la probabilité que cet te espèce colonise les fonds de nos rivières. Les exemplaires déterminés comme $P$. idrensis par Rodriguez (1981) sont en réalité $P$. rosea, qui présente, sur nos exemplaires, une large gamme de formes et proportions dans les dents des aiguilles, quelques-unes très proches de celles de $P$. idrensis.

31. Pristina longiseta Ehrenberg, $1828 \mathrm{n}=84$.

Stations : 7, 10, 11, 12, 14, 34, 35.

Péninsule Ibérique : Rodriguez (1981).

32. Pristina proboscidea Beddard, $1896 n=2$.

Stations : 10, 11.

Péninsule Ibérique : Première donnée.

Habitat : Dans des sédiments caillouteux-sablonneux, dans les eaux claires.

Remarques: Cette donnée confirme la présence de $P$. proboscidea en Europe, considérée jusqu'à main. tenant comme douteuse (Martinez-Ansemil et Giani, sous presse).

Description du matériel : Proboscis court. $n=14$, 16. Sur les faisceaux ventraux de II à VII il y a 2-4 soies. En VIII on remarque une augmentation subite du nombre de soies (jusqu'à 8 par faisceau), celles-ci diminuent de longueur par rapport aux pré. cédentes. La dent supérieure est toujours plus longue que l'inférieure. Dans les faisceaux dorsaux nous trouvons 1 à 3 aiguilles et 1 à 2 grosses soies capillaires. Celles-ci présentent une denticulation évidente : ces denticulations sont espacées de $5 \mu \mathrm{m}$ en moyenne, cet te distance diminue vers le bout de la soie capillaire. Michaelsen a décrit deux formes de cette espèce, d'après la distance entre les denticulations et la longueur des capillaires. En nous référant à ces critères, nos exemplaires appartiennent à la forme typica, qui est celle qui présente la plus petite distance entre les denticulations $16 \mu \mathrm{m}$ environ) et une longueur de capillaires similaire au diamètre du corps. C'est en plus, la forme qui a la distribution géographique la plus ample.

$\begin{array}{crccc}\text { Exemplaire II à VII } & \begin{array}{c}\text { VIIl } \\ \text { à la fin }\end{array} & \text { Aiguilles Capillaires } \\ 1 & 78-80 \mu \mathrm{m} & 58 \mu \mathrm{m} & 45-60 \mu \mathrm{m} & 226-240 \mu \mathrm{m} \\ 2 & 70 \mu \mathrm{m} & 50-60 \mu \mathrm{m} & 46-50 \mu \mathrm{m} & 150-260 \mu \mathrm{m}\end{array}$

\section{- Famille Tubificidae}

33. Tubifex tubifex (Muller, 1774) $n=232$.
Stations : 2, 3, 4, 5, 6, 7, 8, 12, 13, 17, 22, 24, 30, 32, 34. Péninsule Ibérique : Margalef (1955); Prat (1980); Martinez-Ansemil et Giani (1980).

34. Tubifex ignotus (Stolc, 1886) $\mathrm{n}=9$.

Stations: 9, 12.

Péninsule Ibérique : Martinez-Ansemil et Giani (1980). 35. Tubifex costatus (Claparède, 1863) $\mathrm{n}=35$. Station : 15

Péninsule Ibérique : Rodriguez (1981), On la trouve sur presque toute la côte at lantique européenne. La citation de Lasserre (1966) dans la baie d'Arcachon est la plus proche de notre aire d'étude.

36. Limnodrilus claparedeanus Ratzel, $1868 \mathrm{n}=15$.

Stations: 6, 8, 12, 14, 18, 27.

Péninsule Ibérique : Rodriguez (1981).

37. Limnodrilus hoffmeisteri Claparede, $1862 \mathrm{n}=$ 586.

Stations : 4, 5, 6, 7, 8, 10, 12, 13, 14, 19, 24, 25, 31, 34 .

Péninsule Ibérique: Martinez-Ansemil et Giani (1980) : Prat (1980).

38. Limnodrilus profundicola (Verrill, 1871) $\mathrm{n}=30$.

Stations : 3, 14, 31, 32, 34, 36.

Péninsule Ibérique : Rodriguez (1981).

39. Limnodrilus udekemianus Claparède, $1862 \mathrm{n}=$ 37.

Stations: $2,5,6,8,13,14,30,34$.

Péninsule Ibérique : Martinez-Ansemil et Giani (1980).

40. Potamothrix bavaricus (Oschman, 1913) $\mathrm{n}=76$.

Stations: 2, 3, 4, 5.

Péninsule Ibérique : Rodriguez (1981).

41. Potamothrix hammoniensis (Michaelsen, 1901) $\mathrm{n}=63$.

Stations: 2, 3, 5.

Péninsule Ibérique : Prat (1980).

42. Psammonyctides barbatus (Grube, 1861) $\mathrm{n}=166$.

Stations : $2,4,6,7,8,10,11,12,13,14,24$.

Péninsule Ibérique : Martinez-Ansemil et Giani (1980). 43. Ilyodrilus templetoni (Southern, 1909) $\mathrm{n}=1$. Station : 8.

Péninsule Ibérique : Première donnée.

44. Branchiura sowerbyi Beddard, $1892 \mathrm{n}=55$.

Stations: $6,7,8$.

Péninsule Ibérique : Haro (1964); Prat (1980).

45. Aulodrilus limnobius Bretscher, $1899 \mathrm{n}=2$.

Station : 9.

Péninsule Ibérique : Première donnée.

46. Aulodrilus pluriseta Piguet, $1906 \mathrm{n}=2$.

Station : 12.

Péninsule Ibérique : Martinez-Ansemil et Giani (1980).

47. Aulodrilus pigueti Kowalewski, $1914 \mathrm{n}=2$. 
Stations : 6, 24.

Péninsule Ibérique : Première donnée.

48. Bothrioneurum vejdovskyanum Stolc, $1888 \mathrm{n}=$ 195.

Stations : 4, 6, 7, 11, 12, 14 .

Péninsule Ibérique : Martinez-Ansemil et Giani (1980). Remarque : Nous ne l'avons jamais trouvée mature. 49. Rhyacodrilus coccineus (Vejdovsky, 1875) $\mathbf{n}=3$. Stations: 9, 11.

Péninsule Ibérique : Martinez-Ansemil et Giani (1980). 50. Monopylephorus rubroniveus Levinsen, 1884

$\mathrm{n}=29$.

Station: 15.

Péninsule Ibérique : Première donnée.

Remarque : Elle a été citée dans plusieurs localités de la côte atlantique européenne (Kasprzak 1973 b, Goodrich 1895, Ferronière 1899 ; Pfannkuche 1980. Verdonschot 1981).

51. Tubifex sp. $\mathrm{n}=4$ (fig. 3).

Station : 2.

Habitat : Dans un milieu à forte salinité et de pollution organique moyenne. Sur fonds caillouteux.

Description du matériel : Le caractère le plus net de cette forme est la présence de soies géantes sur les faisceaux ventraux des segments III à V. Les mesures que nous donnons ci-dessous appartiennent à 1 exemplaire immature. Soies dorsales : crochets pect inés et soies capillaires. Les crochets antérieurs ont 3 ou 4 dents intermédiaires, les postérieurs aucune. Les soies ventrales sont de 3 types:

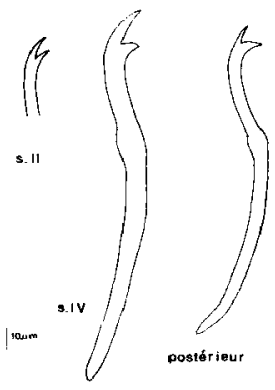

soies ventrales

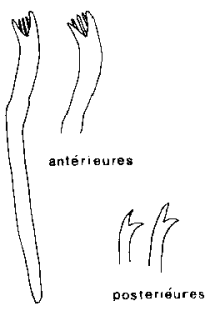

soies dorsales
Fig. 3. Tubifex sp. Crochets dorsaux et ventraux.
1) Les soies de II, avec la dent supérieure plus longue que l'inférieure, nodulus distal, 2 soies par faisceau.

2) Soies géantes en III-V, avec la dent supérieure 2,5 fois plus longue que l'inférieure, nodulus distal, 2 soies par faisceau.

$\begin{array}{ccr}\text { Soies } & \text { Longueur } & \text { Largeur } \\ \text { IV } & 100 \mu \mu \mathrm{m} & 5 \mu \mathrm{m} \\ \text { V } & 110{ }_{\mu} \mathrm{m} & 5 \mu \mathrm{m} \\ \text { VI } & 77,5 \mu \mathrm{m} & 3,7 \mu \mathrm{m}\end{array}$

3) Au-delà de VI, les soies sont normales en longueur $(80 \mu \mathrm{m}$ environ) et largeur $(3 \mu \mathrm{m})$. La dent supérieure diminue peu à peu vers l'arrière jusqu'à devenir égale à l'inférieure, mais un peu plus fine. 1 à 3 soies par faisceau. Nodulus distal. Les individus matures ne possédaient pas de soies génitales. Leur dissection montre que l'appareil génital était, dans un cas, identique à celui de $T u b i$ fex tubifex et que, dans l'autre cas, le " canal éjaculateur " se prolongeait une fois et demi de plus. Cependant, l'union de la prostate et celle du canal déférent à l'atrium étaient caractéristiques du genre Tubifex. Le manque d'exemplaires matures ne nous a pas permis de faire l'identification précise de ce matériel, mais nous pensons qu'il pourrait s'agir d'une variété de l'espèce Tubifex tubifex, à soies géantes, des eaux salées.

\section{- Famille Enchytraeidae}

52. Cernosvitoviella atrata (Bretscher, 1903) $n=16$. Stations: 10, 11, 19.

Péninsule Ibérique : Martinez-Ansemil et Giani (1980). Remarques: Nous avons trouvé les deux formes décrites par Nielsen \& Christensen (1959).

53. Cognettia cognettii (Issel, 1905) $\mathrm{n}=1$.

Station : 9.

Péninsule Ibérique : Martinez-Ansemil et Giani (1980). 54. Cognettia glandulosa (Michaelsen, 1888) $\mathrm{n}=1$. Station : 9.

Péninsule Ibérique : Martinez-Ansemil et Giani (1980.) 55. Lumbricillis kaloensis Nielsen \& Christensen, $1959 \mathbf{n}=124$.

Station : 15.

Péninsule Ibérique : Première donnée.

56. Marionina argenia (Michaelsen, 1889) $n=1$. Station: 12.

Péninsule Ibérique : Martinez-Ansemil et Giani (1980). 
57. Marionina riparia Bretscher, $1889 \mathrm{n}=9$.

Station : 12.

Péninsule Ibérique : Martinez-Ansemil et Giani (1980).

58. Henlea ventriculosa (Udekem, 1854) $\mathrm{n}=1$.

Station : 7 .

Péninsule Ibérique : Rodriguez (1981).

\section{- Famille Lumbrictdae}

59. Eiseniella tetraedra (Savigny, 1826) $n=7$.

Stations: 1, 4, 5, 8, 11, 29, 34.

Péninsule Ibérique : Alvarez (1973); Omodeo (1961); Martinez-Ansemil et Giani (1980).

Remarques : Sur les 7 exemplaires étudiés, 4 sont de la forme typica, 1 de la f. hercynia Michaelsen, et 2 exemplaires sont atypiques, l'un avec une paire de pores mâles en XV et un pore $\sigma$ surnuméraire sur le segment 16 (côté gauche), et l'autre avec un décalage de droite à gauche dans la position des pores et du clitellum (pore masculin : 13, clitellum : 21-25, puberculum : $22-24$ à droite et pore masculin : 12, clitellum : 22-26, puberculum : 23-25 à gauche). Cette forte variabilité dans la position des pores mâles et du clitellum a été soulignée par divers auteurs (Michaelsen 1900, Cernosvitov 1942. Pool 1936, Zajonc 1980). Omedeo (1961), a cité pour la première fois la forme hercynia pour la Péninsule Ibérique, en Navarre, zone proche de notre aire d'étude. Postérieurement, Alvarez (1973) a signalé les formes typica (Savigny) et neapolitana. (Orley).

\section{Travaux cliés}

Alvarez (J.). 1973. - Oligoquetos terrícolas Ibéricos. 2. Lumbricidos (2e pattie). Bol. R. Soc. Espanola Hist. Nat. (Biol.) 71 : 209-211

Alvarez (J.) et Selga (D.). 1968. - Observaciones sobre los inver. tebrados dulceacuícolas de los alrededores de Madrid. Bol. R. Soc Espanola Hist. Naf. (Biof) 65 : 171-197.

Brinkhurst (R.O.) 1978. - Oligochaeta. In : Limnofauna Europaea „J. Illies ed., A. Fischer Verlag, Stuttgart. p. $110-117$.

Brinkhurst (R.O.) el Jamieson (B.G.M.). 1971. - The Aquatic Oligo chaeta of the World. Oliver \& Boyd, Edinburg, p. 860

Cernosvitov (L). 1942. - Oligochaeta from various parts of the World. Proc. Zool. Soc. London 111: 197-236.

De Haro (A.). 1964. - Sobre la distribución de tos oligoquetos en Espana. Branchiura sowerby Beddard, 1892, forma cosmopolita, encontrada en Espana. Bol. R. Soc. Espanola Hist. Nat. (Biol). $62: 137.142$.

Delay (B.). 1973. - Deux nouveaux oligochètes Haplotaxides troglobies d'Espagne: Haplotaxis navarrensis n. sp. et Haplolasis camiabronensis n. $\mathrm{sp}$. Annales Spélèt. 28 : 405-411

Ferronière (G.). 1899.- - III Contribution à l'étude de la faune de la Luire Inférieure (Annelides, Oligochètes). Bull. Soc. Sci. Nat. Ouest Nantes $9: 229-295$.
Giani (N.) et Martinez-Ansemil (E.). 1981. - Coniribution à la connaissance des Oligochètes Aquatiques du Bassin de l'Argens (France). Annts. Limnol. 17 (2) : 121-141.

Goodrich (E.S.). 1892. - Note on a new Oligochaet (Vermiculus pilosus) Zool Anz. XV : 474-476.

Harman (W.J.). 1980. - Specific and generic criteria in freshwater Oligochaeta, with special emphasis on Naididae. In : Aquaric Oligochate Biology. R. O. Brinkhurst \& D. G. Cook Eds. Plenum Press. New York. : $529 \mathrm{p}$.

Hrabe (S.). 1963. - Rhvacodrilus lindbergi n. sp., a new cavernicolous species of the familly Tubificidae (Oligochat ta) from Portugal. Biol. Soc. Portug. Cienc. Nat., 10:52.56.

Hrabe (S.). 1981. - Vodni Malostetinatci (Oligochaeta) cekosloveska. Acla Universilatis Carolinae-Biologica. 1979: 1-167.

Kasprzak (K.) 1973 a. - Nais christinae sp. n., a new species of Naididae (Oligochaeta) found in Poland. Bull. Acad. Pol. Sci. (Ser. Sci. Biol.) 21 (7-8): 535-539.

Kasprzak (K.). 1973 b. - Notaki o faunie Skaposzczetow (Oligochaeta) Polski. J. Fragm. Faun. Warsaw 23:405-434.

Lafont (M.). 1982. - Les Oligochetes des Lacs de Gerardmer ef de Longemer (Departement des Vosges, France). Sciences de leats 1 (1): 21-30.

Lafont (M.) et Juget (J.). 1976. - Les Oligochetes du Rhóne. 1. Relèves faunistiques généraux. Amts. Limmol. 12 : 253-268.

Lasserre (P.). 1966. - Oligochètes marins des côtes de France. I. Bassin d'Arcachon: systématique. Cah. Biol. Mar., 7: 295-317

Loden (M.S.) et Harman (W.J.). 1980. - Ecophenotypic variation in setae of Naididae (Oligochaeta). In : Aquatic Oligochaete Biolog, R. O. Brinkhurst et D. G. Cook Eds. Plenum Press. New York: $\$ 29 \mathrm{n}$.

Margalef (R.). 1935. - Contribución al estudio de las aguas dulces del Noroeste de Espana. P. Inst. Biol. Apl. Barcelona, $21: 137.171$.

Martinez-Ansemil (E.). 1981. - Estudio taxonómico y ecológico comparativo de los oligoquetos del río Tambre (Galicia) y Argens (Sur de Francia). Thèse. Santiago de Compostela. 358 p.

Martinez-Ansemil (E.). 1982. - Les oligochètes aquatiquęs de la Péninsule Ibérique (2 note), avec la description de Lumbricillus brunoi n. Sp. (Enchytraeidae). Soc. Hist. Nat. Toulouse, $118: 145-151$.

Martinez-Ansemil (E.) et Giani (N.). 1980. - Premières données sur les Oligochètes aquatiques de la Péninsule Ibérique. Annls. Limnol. 16 (1): 45-54.

Martinez-Ansemil (E.) et Giani (N.). sous presse. - Cont ribucion al conocimento del genero Pristina (Oligochaeta, Naididae) en la Peninsula Iberica. Bot. R. Soc. Esp. Hist. Nat. Biol., à paraître.

Michaelsen(W.). 1900. - Oligochaeta. In : Das Tierrejch. Berlin : $575 \mathrm{p}$.

Nielsen (C.O.) and Christensen (B.) 1959. - The Enchytraeidae, critical revision and taxonomy of european species. Natura Jutl. $8.9: 1.160$.

Omodeo (P.) 1961. - Oligocheti della Francia meridionale e di localite limitrofe. Mem. Mus. Civ. Storia Nat. Verona $9: 67.95$.

Pfannkuche (O.). 1980. - Aquat ic Oligochaeta (Annelida) from the littoral zone of the Limfjord (Denmark). Verh. Satumiss. Ver. Hamburg. (N.F.) $23: 249.254$.

Pool (G.). 1936. - Eiseniella tetraedra (Sav.). Ein Beitrag zur vergleichenden Anatomie und Systematik der Lumbriciden. Acta Zool. Stockhoim 18 : 1-100.

Prat (N.). 1980. - Bentos de los embalses espanoles. Oecol. Aquatica 4 ; $3-43$.

Rodriguez (P.). 1981. - Primeros resultados del estudio de los oligoquetos acuáticos del Pais Vasco. VReunión Bienal R. Soc. Espanola Hist. Nat: 130

Sperber (C.). 1948. - A Taxonomical study of the Naididae. Zool Bidr. Uppsala 28 : $1-296$. 
Timm (T.). 1979. - The Estonian Lumbriculidae. Eesti NSV Teadoste Akad. Toimetised 28 (Biol.) N. R. 3:158-171.

Tynen (M.M.). 1966. - A new species of Lumbricillus with a revised check-list of the British Enchytraeidae (Oligochaeta). J. Mar. Biol. Ass. U. K. : 89.96 .
Verdonschot (P.F.M.) 1981. - Some notes of ecology of aquatic oligochaetes in the Delta Region of the Netherlands. Arch. Hydrobiol. 92 (1): 53.70 .

Zajone (L.). 1980. - New knowledge on the genus Eiseniella (Lumbricidae). Biologia (Bratislava) 35 (5) : 341-348. 